\title{
Competências para a gestão de um repositório institucional: o caso do repositório institucional da Universidade de Coimbra
}

\author{
Competencies for institutional repositories management: the case of the \\ institutional repository of the University of Coimbra
}

\section{Competencias para la gestión de repositorios institucionales: el caso del repositorio institucional de la Universidad de Coimbra}

\author{
Beatriz Barrocas Ferreira ${ }^{1, a}$ \\ beatrizlbnf.b@hotmail.com | http://orcid.org/0000-0003-4402-9342
}

Bruno Neves ${ }^{2, b}$

bneves@uc.pt| http://orcid.org/o0oo-0002-8669-5514

Ana Miguéis ${ }^{2, b}$

evamigueis@sib.uc.pt | http://orcid.org/o0o0-0003-2869-7754

Maria Manuel Borges ${ }^{1,3, c}$

mmb@fl.uc.pt | http://orcid.org/o000-0002-7755-6168

\footnotetext{
${ }^{1}$ Universidade de Coimbra, Faculdade de Letras. Coimbra, Portugal.

2 Universidade de Coimbra, Biblioteca Geral. Coimbra, Portugal.

3 Universidade de Coimbra, Centro de Estudos Interdisciplinares do Século XX, Faculdade de Letras. Coimbra, Portugal.

a Mestrado em Ciência da Informação pela Universidade de Coimbra.

bestrado em Informação, Comunicação e Novos Media pela Universidade de Coimbra.

c Doutorado em Ciência da Informação pela Universidade de Coimbra.
}

\section{RESUMO}

É num cenário de abertura crescente da ciência, mais amplo e complexo, que se impõem novas competências aos gestores de repositórios. Este estudo visa identificar os papéis e as competências necessárias à gestão e organização de um repositório institucional. Trata-se de um estudo qualitativo com observação participante, suportado pela revisão da literatura, que permitiu refletir sobre o papel dos gestores de repositórios e as competências que devem reunir no âmbito da ciência aberta, tendo como estudo de caso o Estudo Geral, o repositório institucional da Universidade de Coimbra. Embora as funções de um gestor de repositório tenham origem no campo da Ciência da Informação, o conjunto de competências a reunir é amplo e interdisciplinar, destacando-se aquelas ao nível da comunicação científica, das tecnologias digitais, da gestão e da comunicação, da técnica e da gestão de conteúdos.

Palavras-chave: Repositórios institucionais; Gestão de repositórios; Competência; Gestor de repositório; Acesso aberto. 


\begin{abstract}
A scenario of increasing openness of science, which is broader and more complex, requires new competencies of repository managers. This study aims to identify the roles and competencies necessary for the management and organization of an institutional repository. It is a qualitative study with participant observation, supported by the literature review, which allowed us to reflect on the role of repository managers and the skills they must gather in the context of open science, having as a case study the General Study, the repository of the University of Coimbra. Although the functions of a repository manager originate in the field of Information Science, the set of skills to be gathered is broad and interdisciplinary, highlighting the skills in terms of scientific communication, digital technologies, management and communication, technical and content management.
\end{abstract}

Keywords: Institutional repositories; Repository management; Competences; Repository manager; Open access.

\title{
RESUMEN
}

Es en un escenario de creciente apertura de la ciencia, más amplio y complejo, donde se imponen nuevas competencias de los gestores de repositorios. Este estudio tiene como objetivo identificar los roles y competencias necesarios para la gestión y organización de un repositorio institucional. Se trata de un estudio etnográfico, sustentado en la revisión de la literatura, que permitió reflexionar sobre el rol de los gestores de repositorios y las habilidades que deben reunir en el contexto de la ciencia abierta, teniendo como caso de estudio el Estudio General, el repositorio institucional de la Universidad de Coimbra. Si bien las funciones de un gestor de repositorios se originan en el ámbito de la Ciencia de Información, el conjunto de competencias a recopilar es amplio e interdisciplinario, destacando las competencias en materia de comunicación científica, tecnologías digitales, gestión y comunicación, técnica y de gestión de contenidos.

Palabras clave: Repositorios institucionales; Gestión de repositorios; Competencias; Administrador de repositorios; Acceso abierto.

Contribuição dos autores:

Concepção e desenho do estudo: Beatriz Ferreira, Bruno Neves, Ana Miguéis, Maria Manuel Borges.

Aquisição, análise ou interpretação dos dados: Beatriz Ferreira, Bruno Neves, Ana Miguéis.

Redação do manuscrito: Beatriz Ferreira.

Revisão crítica do conteúdo intelectual: Maria Manuel Borges.

Revisão respeitante ao Estudo Geral: Bruno Neves, Ana Miguéis.

Declaração de conflito de interesses: não há.

Fontes de financiamento: não houve.

Considerações éticas: não há.

Agradecimentos/Contribuições adicionais: não há.

Histórico do artigo: submetido: 29 dez. 2021 | aceito: 12 ago. 2021 | publicado: 10 nov. 2021.

Apresentação anterior: não há.

Licença CC BY-NC atribuição não comercial. Com essa licença é permitido acessar, baixar (download), copiar, imprimir, compartilhar, reutilizar e distribuir os artigos, desde que para uso não comercial e com a citação da fonte, conferindo os devidos créditos de autoria e menção à Reciis. Nesses casos, nenhuma permissão é necessária por parte dos autores ou dos editores. 


\section{INTRODUÇÃO}

As Tecnologias de Informação e Comunicação (TIC) oferecem novas possibilidades ao ciclo de comunicação da ciência, tornando os resultados de investigação mais acessíveis por meio da remoção de barreiras ao leitor, tais como permissões de copyright, de licenciamento e de custos de acesso (SUBER, 2012). O Acesso Aberto (AA) constitui uma afirmação da necessidade de aproveitar as oportunidades nascidas da tecnologia digital para recentrar o sistema de comunicação da ciência em torno de partes interessadas como sejam as instituições de ensino superior, as bibliotecas, as agências de financiamento, os investigadores, além da sociedade em geral.

O acesso à informação é uma questão central na forma de fazer e comunicar ciência, visto que é condição para a produção de conhecimento. A Declaração de Budapeste, resultado da Budapest Open Access Initiative (BOAI), tornada pública em 2002, impulsionou e afirmou o movimento do AA. Representa, por essa razão, um dos momentos mais importantes para o AA, já que constituiu o seu sentido e finalidade, apresentando a sua definição e as estratégias para promover e alcançar a publicação em revistas em AA (via dourada) e o auto-arquivo (via verde) (BOAI, 2002).

O movimento do AA fortaleceu-se com o estabelecimento de políticas de AA e com o desenvolvimento de infraestruturas, especialmente os repositórios institucionais ${ }^{1}$ (RI) (KUPRIENE்; PETRAUSKIENE், 2018). Os RI são conjuntos de sistemas e de serviços proporcionados pelas instituições, que têm como finalidade a gestão e disseminação dos conteúdos científicos, por meio de sua recolha, organização, preservação e difusão (LYNCH, 2003; PINFIELD, 2009). Neste sentido, permitem o seu acesso e utilização, não só à comunidade científica e académica, mas também à sociedade em geral. Assim, destacam-se como ferramentas essenciais para as instituições, uma vez que disseminam a sua propriedade intelectual de forma eficaz e eficiente, aumentando a visibilidade e o impacto da investigação e, consequentemente, o prestígio das instituições (SWAN, 2011).

A introdução dos RI no cenário da investigação científica revolucionou o sistema de comunicação das ciências ao introduzir novos papéis para os seus intervenientes. Consequentemente, trouxe várias mudanças e desafios para o ambiente em que se constrói e desenvolve a ciência, onde as bibliotecas, especialmente as académicas e as de investigação, obtiveram um lugar de destaque, assumindo novas funções. Destas, sobressaem as responsabilidades em torno da gestão de repositórios nas suas instituições. Estas funções estimularam o desenvolvimento e a aquisição de um conjunto de competências amplo e diversificado, originando especialidades que deram origem a novas profissões no seio das bibliotecas académicas, nomeadamente a profissão de gestor de repositório (COX; CORRALL, 2013). Tendo em conta o contexto do surgimento dos RI, as competências necessárias à sua gestão incluem um conhecimento aprofundado no domínio da comunicação científica, nomeadamente sobre questões do acesso aberto, entre outros.

Mediante o exposto, pretende-se identificar as competências dos gestores de repositórios na gestão e organização de um repositório institucional, tendo como estudo de caso o repositório institucional da Universidade de Coimbra (UC) - o Estudo Geral.

O Estudo Geral entrou em funcionamento em 2008 inaugurando a presença da UC no AA. Sua missão é aumentar a visibilidade da comunidade científica da universidade e contribuir para a ampliação do impacto da investigação e do prestígio da UC, por meio da recolha, preservação, disseminação e acesso dos conteúdos digitais produzidos pela sua comunidade. Desta forma, o Estudo Geral reúne num só local a produção

\footnotetext{
1 A pertinência do movimento dos repositórios digitais, inspirados pelo ArXiv e iniciado pela Open Archives Initiative (OAI), associada à relevância das práticas sugeridas pelas declarações de Budapeste, Bethesda e Berlim, confere aos repositórios de AA um lugar de destaque no estabelecimento da via verde. Após a OAI, e com o desenvolvimento do protocolo OAI-PMH, as instituições começaram a desenvolver os seus próprios repositórios e a partilharem os conteúdos em repositórios agregadores, o que fortaleceu o crescimento de repositórios, mas também a abertura da ciência.
} 
científica da UC, agregando os artefactos digitais e permitindo a sua gestão e disseminação para um acesso facilitado e transparente, não só à comunidade científica, mas também à sociedade em geral.

\section{ABORDAGEM METODOLÓGICA}

O presente trabalho tem como foco principal o estudo dos RI, enquanto sistemas de informação que desempenham funções basilares à comunicação científica, e as competências que os gestores encarregues pela sua organização e gestão devem possuir, de forma a cumprir a missão e o propósito do RI. Como objetivos específicos, propõe-se identificar as funções desempenhadas na gestão de um RI; indicar as competências essenciais à sua gestão eficiente e eficaz; e caracterizar as funções e aptidões necessárias à gestão do Estudo Geral.

Foi adotada uma abordagem qualitativa com vista à identificação das competências necessárias à gestão de um repositório institucional, suportada pela revisão da literatura científica e por um estudo de caso com observação participante. Portanto, o estudo compreende duas fases: uma parte teórica para ilustrar a temática em volta das competências para a gestão de RI, e outra, de caráter empírico, que visa a apresentar o caso do Estudo Geral como testemunho do processo para trabalhos futuros.

A revisão da literatura fez-se a dois níveis: primeiramente, procedeu-se à seleção dos artigos recuperados nas bases de dados e catálogo bibliográfico, seguindo para uma análise da pertinência dos artigos por meio da leitura dos títulos, resumos e palavras-chave apresentados nas bases de dados. Esta análise permitiu reduzir os resultados apresentados para os números exibidos na Tabela 1. A leitura aprofundada dos trabalhos, incluindo a sua bibliografia, alargou para um conjunto de artigos não integrados nos resultados de pesquisa realizados nas bases de dados, posteriormente selecionados e recuperados para inclusão no presente estudo.

A pesquisa de informação foi realizada na base de dados Web of Science (WoS), na plataforma Repositórios Científicos de Acesso Aberto de Portugal (RCAAP) e no catálogo integrado das bibliotecas da UC. A escolha da WoS baseou-se no facto de se tratar de uma base de dados cuja abrangência permite realizar uma cartografia ampla da literatura científica, mas também pelas possibilidades de navegação e seleção que oferece para a avaliação da informação recuperada. A escolha do RCAAP fundamenta-se no facto de ser um metarepositório que integra os repositórios científicos de Portugal e também o portal agregador OASISbr. A utilização do catálogo bibliográfico da UC serviu para recuperar monografias sobre repositórios institucionais, consideradas pertinentes para o desenvolvimento do estudo.

$\mathrm{Na}$ recuperação da informação foram utilizados os termos ("gestor de repositório/gestores de repositórios"; "repositório institucional”; "competências") em inglês na WoS, e em inglês e em português nas restantes fontes de informação selecionadas. Contudo, as pesquisas realizadas em inglês nas plataformas RCAAP e catálogo integrado da UC não revelaram resultados, conforme visível na Tabela 1. As pesquisas avançadas foram executadas por assunto, com recurso a aspas e a truncatura, conjugando os termos da equação por meio do operador booleano AND. 
Tabela 1 - Número de artigos recuperados nas fontes de informação selecionadas

\begin{tabular}{|c|c|c|c|}
\hline Fonte de informação & Equação de pesquisa & $\begin{array}{l}\text { Total de } \\
\text { resultados }\end{array}$ & $\begin{array}{l}\text { Resultados } \\
\text { recuperados }\end{array}$ \\
\hline \multirow{3}{*}{ Web of Science } & $\begin{array}{l}\mathrm{TS}=(\text { "institutional repositor*" AND } \\
\text { "repository manager") }\end{array}$ & 4 & 4 \\
\hline & $\begin{array}{l}\text { TS=("institutional repositor*" AND } \\
\text { competencies) }\end{array}$ & 8 & 2 \\
\hline & $\begin{array}{l}\text { TS=("repository manage*" AND } \\
\text { "institutional repositor*") }\end{array}$ & 29 & 7 \\
\hline $\begin{array}{l}\text { Catálogo integrado das } \\
\text { bibliotecas da UC }\end{array}$ & "repositório institucional" & 3 & 1 \\
\hline \multirow{2}{*}{ RCAAP } & "gestores de repositórios" & 4 & 1 \\
\hline & "repository manager+" & 2 & 0 \\
\hline
\end{tabular}

Fonte: elaboração dos autores.

A parte empírica foi sustentada por um estudo de caso, aplicando a observação participante na Biblioteca Geral da UC, no serviço que tem a seu cargo a gestão do Estudo Geral² ${ }^{2}$ captando, assim, algumas experiências decorrentes do contacto direto com a plataforma. Simultaneamente, possibilitou processos sociais e de interação com a equipa de gestão do repositório, o que enriqueceu a recolha das informações relevantes para o desenvolvimento do estudo. $O$ trabalho de campo permitiu a execução de atividades que visam a demonstrar a exigência e complexidade associadas às tarefas de gestão do repositório analisado, proporcionando maior representatividade e impacto da produção científica dos autores e investigadores da UC. As atividades desenvolvidas incidiram, sobretudo, na recolha e depósito de teses de doutoramento e de artigos científicos (migração das teses defendidas e disponíveis na plataforma Provas Académicas; da adição de metadados em falta e revisão/alteração de metadados existentes; da atribuição de "direitos de acesso" às teses; e do seu depósito), na curadoria dos conteúdos depositados (validação e correção de metadados; mapeamento/associação de registos a diferentes coleções; e deteção e eliminação de registos em duplicado), e na configuração de perfis de investigador e no controlo de autoridade (levantamento e identificação dos investigadores da UC no Estudo Geral; atualização de perfis de investigador e associação de outros identificadores; desambiguação de perfis de investigadores e associação da respetiva produção científica).

\section{O GESTOR DE UM REPOSITÓRIO INSTITUCIONAL (RI)}

Os RI, enquanto infraestruturas que permitem a organização, preservação e disseminação da informação (PINFIELD, 2009), facilitam o acesso aos conteúdos científicos, de diversas tipologias. Os RI contribuem para o aumento da visibilidade, prestígio e impacto da investigação, não só dos investigadores como também da própria instituição, alinhando-se com os requisitos de órgãos externos, como sejam as agências de financiamento de ciência (RUMSEY, 2006). No caso das instituições de ensino superior (IES), as bibliotecas académicas e de investigação são as responsáveis pelo desenvolvimento e gestão dos seus repositórios, o que inclui responsabilidades de organização, gestão e disseminação da informação e de preservação, além dos papéis de mediadoras da informação e de promoção do AA. Os RI surgem, então, como uma das respostas à comunicação científica na era digital, fomentando novas práticas e técnicas

2 Anteriormente designado Serviço Integrado das Bibliotecas da Universidade de Coimbra. 
científicas. Consequentemente, a sua implementação desencadeou novas funções no seio das bibliotecas académicas e de investigação, exigindo também novas aptidões e competências aos responsáveis pela sua gestão (SIMONS; RICHARDSON, 2012).

As bibliotecas detêm um papel central na investigação científica, e, numa esfera em constante mudança e cada vez mais digital, veem-se pressionadas em se reinventar para continuar a manter o seu papel relevante junto das suas comunidades. O ambiente da abertura crescente da ciência desencadeia assim uma série de desafios às bibliotecas, mas, ao mesmo tempo, estimula também o surgimento de novas oportunidades. O aproveitamento das possibilidades oferecidas pelas TIC, a introdução de ferramentas e plataformas digitais (como a implementação de RI nas suas instituições), assim como a elaboração e execução de boas práticas de investigação e de políticas de AA, manifestam-se como algumas destas oportunidades para as bibliotecas (CASSELA; MORANDO, 2012). É neste seguimento que os papéis tradicionais das bibliotecas, como a gestão e disseminação de coleções ou o serviço de referência, se inovam e atualizam, levando ao aparecimento de novas especialidades e profissões no seio das bibliotecas das IES e, consequentemente, novas funções que estimulam o desenvolvimento e a aquisição de novas competências.

Surge, também, nesse contexto a profissão de gestor de repositório, oriunda do campo da Biblioteconomia e Ciência da Informação (ZUCCALA; OPPENHEIM; DHIENSA, 2008) e, por conseguinte, com um carácter interdisciplinar intrínseco. Assim sendo, a profissão de gestor de RI exige um conjunto de competências específicas provenientes de diferentes áreas do conhecimento, mas ao mesmo tempo convergentes. Desse modo, embora as funções tenham origem em contexto biblioteconómico, excedem os conhecimentos tradicionais desempenhados pelos bibliotecários (SIMONS; RICHARDSON, 2012). Por conseguinte, as contribuições dos gestores são reestabelecidas e reinventadas.

O rápido crescimento dos RIs, sobretudo em contexto académico, estimula a constante atualização de conhecimentos, de aptidões e de competências, por parte dos elementos envolvidos na gestão de um RI. Embora o desenvolvimento, manutenção e gestão de um RI englobe vários elementos com responsabilidades diferentes - nomeadamente membros dos sistemas e tecnologias de informação; bibliotecários de sistemas de metadados e de referência; técnicos e administrativos; e administrador do RI -, o gestor de RI merece um papel de destaque por ser o elemento encarregue pela componente humana do repositório (SHERPA, 2009). Esta componente compreende a gestão de políticas relativas ao conteúdo, a promoção e divulgação do RI e do AA, apoio e formação de utilizadores e comunicação com outros órgãos institucionais e externos (SHERPA, 2009). O gestor de RI tem assim um papel essencial no cumprimento dos objetivos e no sucesso do repositório. Não obstante, a equipa de suporte à gestão e administração do RI demonstra-se essencial, reinvindicando a existência de uma equipa completa e interdisciplinar, constituída por especialistas em informação e computação (ZUCCALA; OPPENHEIM; DHIENSA, 2008). Porém, existem várias instituições científicas que não refletem esta realidade, apresentando equipas com poucos elementos que prejudicam o desenvolvimento e o sucesso dos seus RIs (SALO, 2008).

Existem várias áreas de atuação no que concerne ao desenvolvimento e disponibilização de um RI. Wickham (2010), num estudo desenvolvido sobre repositórios institucionais no Reino Unido, identifica três práticas essenciais: a gestão de RIs; a componente técnica e a administrativa, em que a gestão de repositórios envolve "strategic and financial management, advocacy and communication, staff and project management, expert advice to the institution" (WICKHAM, 2010, p. 5). Nesta lógica, foi lançado um documento SHERPA em 2007, revisto em 2009, que identifica dois cargos fundamentais envolvidos na gestão de RIs: o gestor de repositório e o administrador de repositório (SHERPA, 2009), em que o administrador de RI coordena a implementação técnica do repositório, nomeadamente questões relacionadas com o software, a preservação de conteúdos e gestão de metadados, enquanto o gestor se responsabiliza pelo planeamento, gestão de políticas, planos de comunicação e contactos com a comunidade 
interna e externa. Deduz-se então que as funções de um gestor de RI consistem no planeamento e gestão de um serviço eficaz e eficiente, contribuindo para a satisfação das necessidades da sua comunidade e para o cumprimento da missão da sua instituição.

Dado o surgimento dos repositórios num contexto em que o crescimento das tecnologias digitais, ampliadas pela Internet e pela World Wide Web, modificou o modo como o conhecimento é produzido e disseminado, contribuindo para a consolidação do AA e fortalecimento da CA, destacam-se alguns conhecimentos no cenário da comunicação científica essenciais para o desempenho da função de gestor de RI (THOMAS, 2013). Portanto, o conhecimento do ciclo de investigação e comunicação científica (nomeadamente as diversas fases do ciclo e o sistema de publicação científica, assim como os diversos canais e formas de comunicação possíveis e a anatomia de um repositório digital científico), a proximidade com princípios e políticas de AA (de agências financiadoras de ciência, instituições de referência e da própria instituição), e a familiaridade com os conceitos defendidos pela CA são algumas das aptidões que o gestor de RI deve reunir no que concerne às competências de comunicação científica (THOMAS, 2013). Inerentemente, por ser também uma função originária da Biblioteconomia e da Ciência da Informação, exige o domínio de competências biblioteconómicas que envolvem vários fatores. Neste seguimento, Cassela e Morando (2012) identificam outras cinco competências basilares que o gestor deve reunir, relacionando-se com as biblioteconómicas acima referidas:

a) competências no domínio da comunicação e promoção;

b) competências no domínio da gestão;

c) competências relativas a questões técnicas;

d) competências no domínio da propriedade intelectual;

e) competências no domínio da gestão de coleções e metadados.

Zuccala, Oppenheim e Dhiensa (2008) desenvolveram um estudo que se debruçou sobre a elaboração de um currículo para gestores de repositórios, onde destacam também cinco componentes fundamentais para as funções de gestão de um repositório. Salientam assim:

a) conhecimentos acerca do ambiente de publicação digital e de investigação científica, marcado pela sua constante mudança, e onde realçam questões relativas à comunicação científica, como os desenvolvimentos de publicação eletrónica, princípios e modelos de AA, entre outros;

b) conhecimento sobre repositórios digitais, incluindo os diversos tipos e tendências atuais de investigação sobre o tema;

c) conhecimentos de gestão, nomeadamente gestão financeira, de recursos humanos, marketing e de estatísticas de uso;

d) conhecimentos biblioteconómicos, especialmente sobre bibliotecas digitais e desenvolvimentos recentes em biblioteconomia;

e) conhecimentos acerca de ferramentas técnicas, relacionados com as funções técnico-administrativas levadas a cabo durante a gestão de repositórios.

Mediante o exposto, conclui-se que a função de gestor de RI exige competências em diversos domínios, dada a natureza interdisciplinar de um repositório digital e da constante mudança do ambiente em que ele se insere. Para além das competências supracitadas, o gestor do RI deve ser o elemento que define e reconhece de imediato a raison-d'être do repositório, servindo de promotor e guia na sua divulgação e utilização junto da sua comunidade (ZUCCALA; OPPENHEIM; DHIENSA, 2008). Realça-se também a importância e a necessidade de formação contínua e ao longo da vida do gestor, de forma a manter atualizados os seus conhecimentos e enriquecer as suas aptidões e desempenho profissional. 


\section{O ESTUDO GERAL}

Desde que foi inaugurado em 2008, o Estudo Geral (EG) procurou atender às necessidades sentidas quer pela instituição, quer pelos seus investigadores. O software usado para este RI é o DSpace, uma plataforma de código aberto, cuja versão mais recente permitiu, em 2018, a implementação do sistema DSpace-CRIS. As sucessivas atualizações foram introduzindo novas funcionalidades que obrigaram os seus gestores a incorporar um conjunto crescente de funções e atividades. Estas abrangem tarefas que vão desde a implementação e configuração até a preservação dos conteúdos depositados, entre outras, preparando-os para enfrentar os novos desafios oriundos da Ciência Aberta.

A relevância que o repositório foi ganhando junto da comunidade científica da universidade e das suas unidades de investigação justificou que se avançasse para uma solução tecnológica que permite dispor não apenas de um repositório de publicações, mas também, associado a este serviço, de um conjunto de informação sobre a investigação feita na UC, que se traduz num sistema de gestão de informação científica da UC, um CRIS (Current Research Information System). Este sistema, ambicionado desde há algum tempo, que procura interligar as principais componentes que intervêm na investigação - publicações, investigadores, unidades de investigação e projetos - começou a ganhar forma com a implementação do DSpace-CRIS, que permitiu estruturar e incorporar na plataforma informação relevante relacionada com a atividade científica, como seja a informação sobre as unidades de investigação, perfis de investigadores associados ou projetos desenvolvidos (MIGUÉIS; NEVES, 2021).

A solução adotada para o EG possibilita aos gestores do repositório gerir a informação de investigação e, por este meio, promover o acesso e a disseminação da atividade científica realizada na UC, contendo novos elementos que reforçam o impacto da publicação, nomeadamente a disponibilização de estatísticas. A título de exemplo, destaca-se a informação sobre os investigadores que têm no seu 'Perfil do Investigador' dados de identificação (nome, afiliação, identificadores, como o ORCID ou outros, e áreas científicas), mas também indicadores e métricas sobre número de visualizações e downloads das publicações, dados bibliométricos, que incluem número de trabalhos indexados, número de citações na Scopus e na Web of Science e dados sobre métricas alternativas. A implementação do DSpace-CRIS representa um passo importante para a satisfação das necessidades das unidades de investigação da UC ao facilitar a gestão e interligação de várias componentes da atividade científica. Por outro lado, permite beneficiar dos avanços realizados em diversos aspetos, nomeadamente na curadoria digital, no cumprimento das políticas de Acesso Aberto, no desenvolvimento de fluxos de trabalho entre serviços e na interoperabilidade entre sistemas com o recurso a APIs (MIGUÉIS; NEVES, 2021).

Atualmente, o EG representa um importante instrumento para a preservação da memória intelectual da UC e sua projeção, mas é previsível que se venha a converter no sistema de informação de referência para toda a produção científica da UC, com ligação à componente de avaliação dos docentes e investigadores e a validação das agências de financiamento. É neste contexto que os gestores do repositório desempenham um papel cada vez mais central nos processos da gestão da Ciência.

Quanto à sua estrutura, o EG está organizado por comunidades que refletem a composição orgânica da UC, representando as faculdades e as unidades de extensão e de investigação que compõem a universidade. Por seu turno, essas dividem-se em subcomunidades (departamentos) e em secções. As comunidades podem reunir as suas publicações em diferentes coleções, que, por sua vez, podem apresentar um vasto número de documentos e de tipologia documental variada. Assim sendo, o conjunto de documentos reunidos no EG representa conteúdos digitais resultantes das atividades de investigação científica e de ensino desenvolvidas pela comunidade científica e académica da UC. 


\section{A VISÃO DO ESTUDO GERAL NA QUESTÃO DAS COMPETÊNCIAS NECESSÁRIAS À SUA GESTÃO}

A gestão de um RI envolve vários elementos e funções que podem variar de instituição para instituição, embora se destaque o seu planeamento, a promoção do repositório e de boas práticas de comunicação científica, o desenvolvimento e adoção de políticas de AA etc. Dada a volatilidade do ambiente em que se inserem, os repositórios necessitam de uma avaliação contínua que determine novas condições e direções para se desenvolverem, e para determinar a sua qualidade (ZUCCALA; OPPENHEIM; DHIENSA, 2008). A implementação do DSpace CRIS veio aumentar o conjunto de operações que determinam um conjunto diversificado de tarefas, revelando um grau de complexidade que exige competências específicas à sua execução. Os procedimentos que envolvem a administração de um repositório são fundamentais à sua gestão e suporte, mas também à sua sustentabilidade enquanto serviço (JONES; ANDREW; MACCOLL, 2006). Portanto, de modo a identificar as competências necessárias que o gestor do EG deve reunir, é necessário primeiro indicar as tarefas desempenhadas na sua gestão.

A configuração do sistema DSpace-CRIS, como já referido, contribuiu para atender às necessidades sentidas pela comunidade da UC, principalmente das unidades de investigação e desenvolvimento e dos seus investigadores. Ao integrar um sistema de gestão de ciência, o EG possibilita não só o acesso a publicações científicas como também a informação sobre as atividades de investigação realizadas na UC. A integração de informações acerca das atividades de investigação levadas a cabo pela UC e a sua consequente validação enriquecem o EG, tornando-o muito mais do que um simples repositório de publicações científicas.

A estrutura administrativa do EG - o workflow - representa um conjunto de estágios essenciais na gestão do repositório, uma vez que reflete o core do repositório. O workflow de um repositório compreende quatro áreas que podem envolver vários elementos no desempenho das suas tarefas, incluindo as fases de submissão, pós-submissão, preservação e gestão estrutural (JONES; ANDREW; MACCOLL, 2006; ZUCCALA; OPPENHEIM; DHIENSA, 2008).

A gestão administrativa e técnica do EG envolve então essas áreas, tendo início com a fase de submissão, caracterizada pelos procedimentos de aquisição de conteúdo. Essa fase pode ser realizada não apenas pela equipa de gestão do repositório, como também pelos membros/autores da comunidade académica da universidade e é de extrema relevância, já que para o sucesso e cumprimento da missão do RI é necessário que este apresente uma coleção rica e completa. Segue-se a fase de pós-submissão, na qual são realizados os procedimentos relacionados com a curadoria dos conteúdos depositados, envolvendo a verificação, validação e enriquecimento dos metadados; o cumprimento das políticas de copyright dos documentos; o controlo de autoridade (de autor); o mapeamento/ligação de registos a outras coleções; e a deteção e a eliminação de duplicados. A fase de preservação envolve procedimentos relacionados com o armazenamento e preservação dos conteúdos, compreendendo o cumprimento de normas e diretrizes de referência, mas também a verificação da integridade dos ficheiros. Por fim, a fase de gestão estrutural, que abrange a gestão de utilizadores e as suas permissões, a gestão de políticas, a estruturação do arquivo e a gestão dos conteúdos.

A atualização para o DSpace-CRIS, para além de novas oportunidades, trouxe também responsabilidades acrescidas, pelo que a integração de elementos relacionados com as atividades de investigação da UC é uma tarefa bem relevante na gestão do EG. Neste sentido, para além da integração das publicações na coleção do repositório, são também criados e preenchidos os perfis de investigador com informações de afiliação, de contacto e de identificadores únicos digitais; a associação dos perfis aos centros de investigação da Universidade; a identificação de projetos de financiamento às publicações; e a associação de publicações aos devidos autores e, consequentemente, aos centros de investigação a que pertencem. Existe, então, uma forte 
componente de curadoria digital associada a essas tarefas que envolve não só o preenchimento dos elementos das atividades de investigação, como também o controlo de autoridades e a gestão de coleções e metadados.

O tráfego, a utilização e o depósito de documentos são essenciais para a sustentabilidade do repositório e para o cumprimento da sua missão. Uma das estratégias adotadas passou pelo desenvolvimento e implementação da Política de Acesso Livre da Universidade de Coimbra, lançada em 2010. Posto isto, uma função crucial na gestão do RI inclui a certificação do cumprimento não apenas da política da universidade, mas também das políticas emitidas pelas principais agências de financiamento em Portugal, nomeadamente a Fundação para a Ciência e a Tecnologia, e a União Europeia, que lançaram políticas de AA bastante relevantes e de carácter compulsório, determinando o AA às publicações e dados científicos e o seu depósito em repositórios da rede RCAAP. Portanto, os gestores do EG executam também funções que visam ao cumprimento de políticas de AA, fundamentais para aumentar a visibilidade e o impacto da investigação científica e da própria instituição, pelo que é necessária a familiaridade com as diversas políticas existentes e em vigor.

A divulgação e promoção do EG e dos serviços que este disponibiliza à comunidade são tarefas imprescindíveis na definição de estratégias para a gestão do repositório, incluindo o incentivo ao autoarquivo; a comunicação e disseminação das potencialidades do EG junto da sua comunidade; ou a formação de utilizadores no âmbito da comunicação científica. Destaca-se a necessidade de formação contínua dos elementos da equipa de gestão, realçando a presença em conferências e grupos de trabalho no âmbito do AA e RI, nos quais é aproveitada a atualização e partilha de conhecimentos, e dinamizado o EG junto de outras comunidades e potenciais utilizadores. Também o contínuo planeamento e avaliação representam responsabilidades que o gestor deve ter bem presentes e delineadas para o sucesso e valorização do EG. Destaca-se ainda a gestão de recursos financeiros em torno do desenvolvimento do repositório, assim como a gestão de recursos materiais e físicos, e a gestão de recursos humanos.

Os RIs, para além de contribuírem para a consolidação e facilitação do AA, representam uma ferramenta elementar à comunicação científica e práticas de investigação, concorrendo para o aumento da visibilidade e do impacto dos outputs de investigação produzidos pelas suas instituições, e preservando os seus conteúdos. Para que alcancem o seu sucesso e cumpram a sua missão, devem possuir uma estrutura sólida; um planeamento apropriado; uma política institucional de AA (de preferência de carácter compulsório); e, especialmente, uma equipa de suporte à sua manutenção e gestão, constituída por elementos especialistas em informação e informática ( $\mathrm{LYNCH}, 2003$ ), onde se destaca o gestor de repositório, enquanto elemento de coordenação. Contudo, note-se a questão da falta de elementos em equipas de gestão de repositórios institucionais, que provoca lacunas no desenvolvimento dos repositórios e, consequentemente, no cumprimento da sua missão (SALO, 2008).

Dada a constante mudança do ambiente em que os RIs se inserem, caracterizado pelo meio digital e pela abertura crescente da ciência, bem como pela sua interdisciplinaridade intrínseca, o seu gestor deve possuir um currículo alargado e em atualização contínua em várias áreas de atuação, como Biblioteconomia, Ciência da Informação e Tecnologias de Comunicação e Informação. Neste sentido, as competências que deve reunir encontram-se em várias áreas de atuação.

Realça-se a necessidade de reunir conhecimentos aprofundados acerca da anatomia de um repositório digital e do movimento em que estes se enquadram, especialmente no seio da sua instituição. Em conclusão, deve possuir competências:

1. ao nível técnico e administrativo, revelando entendimento relativo à manutenção e à administração do RI, e, por conseguinte, conhecimentos acerca do software implementado; de ferramentas digitais; de normas e de protocolos de interoperabilidade e de preservação digital; de diretrizes à gestão de repositórios; dos procedimentos de workflow e da implementação de serviços de valor acrescentado; 
2. ao nível da comunicação e marketing, nomeadamente aptidões para a elaboração de uma estratégia de promoção do RI, que contribua para a sua utilização e sucesso; para o desenvolvimento de um serviço de apoio e formação à comunidade académica da sua instituição; e para a comunicação frequente com a comunidade interna, designadamente utilizadores, investigadores, centros de investigação e órgãos de direção da instituição, mas também com a comunidade externa, de forma a estabelecer e fortalecer ligações;

3. ao nível da gestão, particularmente relacionadas com a gestão de recursos financeiros, humanos e técnico-administrativos, destacando-se a importância de aptidões de trabalho colaborativo e de gestão de projetos;

4. ao nível biblioteconómico, o que integra a gestão de coleções e de metadados; e curadoria digital;

5. ao nível da propriedade intelectual e outras questões legais, compreendendo conhecimentos ao nível dos direitos de autor, termos de copyright e licenciamento;

6. ao nível da comunicação científica, exigindo conhecimentos aprofundados sobre o movimento de AA e os princípios da CA; sobre políticas de AA existentes, tanto das principais agências de financiamento, como de instituições de referência em investigação científica; sobre o ambiente atual de comunicação científica, das vias de concretização ao AA, dos modelos de publicação eletrónica e sistemas de publicação científica; sobre práticas e ciclo de investigação; e sobre a realidade e linhas correntes de investigação no âmbito dos repositórios científicos digitais e todas as questões envolvidas em seu torno.

Especificamente ao EG, dada a particularidade do software implementado, o gestor deve reunir o conjunto de competências supracitadas e em especial, competências ao nível dos sistemas CRIS, incluindo entendimento sobre normas e diretrizes relativas a este tipo de sistemas (como a norma de exportação de dados CERIF); competências ao nível de identificadores persistentes e a sua relevância no contexto da CA; e competências ao nível de indicadores bibliométricos, incluindo as altmetrias, e outras estatísticas de uso e avaliação da investigação. Mediante o exposto, entende-se que o gestor do EG, para além de desempenhar tarefas comuns na gestão de um RI (embora se saliente que os procedimentos adotados podem variar de instituição), efetiva também funções relacionadas com a gestão de um sistema CRIS.

\section{CONSIDERAÇÕES FINAIS}

Opresente estudo teve como objetivo principal identificar as competências necessárias para o desempenho da função de gestor de RI, adotando como caso de estudo o repositório científico da UC. Por meio da revisão da literatura, identificaram-se algumas competências mencionadas noutros estudos, embora se tenha verificado que não existem trabalhos muito alargados com foco nos gestores de repositórios, nas funções que estes desempenham e nas competências que devem possuir.

Conclui-se que a gestão de repositórios científicos digitais é um processo complexo, que compreende várias componentes e procedimentos em diversas áreas do conhecimento, o que exige competências específicas aos seus gestores. O EG revela-se um instrumento fundamental não apenas à comunicação científica, como à gestão das atividades de investigação da UC, ao recolher, preservar, disseminar e facilitar o acesso à produção científica da Universidade. Ao mesmo tempo, faculta a gestão e a integração de vários constituintes das suas atividades de investigação, contribuindo para a satisfação das necessidades da comunidade académica e para a valorização do repositório e projeção da Universidade de Coimbra.

Por fim, realça-se a pertinência do tema e a importância da partilha de um estudo de caso como testemunho do processo, contribuindo para a construção de processos de recrutamento que envolvam a profissão de gestor de repositório institucional, concorrendo também para o auxílio no desenvolvimento e 
capacitação da profissão. Deste modo, destaca-se a sua relevância para a prática das funções de gestor de repositório, representando um contributo para o desempenho deste papel.

O estudo apresenta algumas limitações, sobretudo ao nível metodológico. Como futuras linhas de investigação, procurar-se-á aplicar métodos de recolha de dados mais abrangentes, como sejam a aplicação de inquérito por questionário ou por entrevista a gestores de repositórios, complementando as informações recolhidas no presente trabalho.

O gestor de repositório tem um papel central à comunicação científica ao ser o responsável pelo estabelecimento e pela gestão de um repositório institucional. Portanto, revela-se um agente essencial na facilitação do conhecimento científico, mas também no suporte à comunicação científica no cenário da ciência aberta. Deste modo, existem várias temáticas pertinentes em torno do seu papel e das responsabilidades exigidas para a gestão eficaz e eficiente de um repositório no contexto atual da investigação científica, cada vez mais caracterizada pelos meios digitais. Num cenário de abertura crescente da ciência, os gestores de RI centram-se no contexto da ciência aberta, trabalhando na implementação dos seus princípios junto das suas comunidades. O Estudo Geral foca-se em enfrentar os desafios desencadeados pela ciência aberta, ao servir como um instrumento central à investigação, mas também à gestão da informação científica.

Em conclusão, recomenda-se a aquisição das competências identificadas para o desenho do perfil de um gestor de RI e também para o desempenho das suas funções. Considera-se relevante que se tome em consideração que as atividades de um gestor de RI se desenvolvam em torno de vários campos de atuação, nomeadamente da gestão de recursos técnicos, humanos e financeiros, mas também da gestão de projetos, da gestão documental e da gestão da informação e comunicação científica. Deste modo, para além da construção de competências nestes domínios, é essencial a atualização de conhecimentos e aptidões, frisando-se a importância da formação contínua e ao longo da vida.

Relativamente ao gestor do Estudo Geral, salienta-se a adoção de competências ao nível da gestão da informação científica, sobretudo ao nível dos sistemas CRIS. O Estudo Geral, ao atualizar o software Dspace-CRIS, dinamizou o conjunto de serviços que este oferece, impulsionando a utilização e relevância do RI no seio da sua comunidade. Neste sentido, a aquisição de competências nesta linha pelo seu gestor contribui não só para a agilização das necessidades dos seus investigadores, mas também para a articulação entre a prática profissional e a de investigação.

\section{REFERÊNCIAS}

BUDAPEST Open Access Initiative. Budapest: OSI, 2002. https://www.budapestopenaccessinitiative.org/ translations/portuguese-translation

CASSELA, Maria; MORANDO, Maddalena. Fostering new roles for librarians: skills set for repository managers - results of a survey in Italy. Liber Quarterly, [s. I.], v. 21, n. 3/4, p. 407-428, 2012. DOI: http://doi.org/10.18352/ la.8033. Disponível em: https://liberquarterly.eu/article/view/10626. Acesso em: 16 set. 2021.

COX, Andrew M.; CORRALL, Sheila. Evolving academic library specialties. Journal of the American Society for Information Science and Technology, [s. I.], v. 64, n. 8, p. 1526-1542, ago. 2013. DOI: https://doi. org/10.1002/asi.22847. Disponível em: https://onlinelibrary.wiley.com/doi/10.1002/asi.22847. Acesso em: 15 set. 2021.

JONES, Richard; ANDREW, Theo; MACCOLL, John. The institutional repository. Oxford: Chandos Pub, 2006.

KUPRIENĖ, Jūratė; PETRAUSKIENĖ, Žibutè. Opening science with institutional repository: a case study of Vilnius University Library. Liber Quarterly, [s. I.], v. 28, n. 1, p. 1-24, out. 2018. DOI: http://doi.org/10.18352/ lq.10217. Disponível em: https://liberquarterly.eu/article/view/10825. Acesso em: 15 set. 2021. 
LYNCH, Clifford A. Institutional repositories: essential infrastructure for scholarship in the Digital Age. portal: Libraries and the Academy, Baltimore, v. 3, n. 2, p. 327-336, 2003. DOI: http://dx.doi.org/10.1353/ pla.2003.0039. Disponível em: https://muse.jhu.edu/article/42865. Acesso em: 15 set. 2021.

MIGUÉIS, Ana Eva; NEVES, Bruno. A visão dos gestores de repositórios: o caso da Universidade de Coimbra. In: BORGES, Maria Manuel; CASADO, Elias Sanz (coord.). Sob a lente da Ciência Aberta: olhares de Portugal, Espanha e Brasil. Coimbra: Imprensa da Universidade de Coimbra, 2021. p. 273-294. DOI: https:// doi.org/10.14195/978-989-26-2022-0 10. Disponível em: http://hdl.handle.net/10316/92886. Acesso em: 16 set. 2021.

PINFIELD, Stephen. Journals and repositories: an evolving relationship?. Learned Publishing, v. 22, n. 3 , p. 165-175, 2009. DOI: https://doi.org/10.1087/2009302. Disponível em: https://onlinelibrary.wiley.com/doi/ abs/10.1087/2009302. Acesso em: 15 set. 2021.

RUMSEY, Sally. The purpose of institutional repositories in UK higher education: A repository manager's view. International Journal of Information Management, [s. I.], v. 26, n. 3, p. 181-186, jun. 2006. DOI: http://dx.doi. org/10.1016/j.jijinfomgt.2006.01.002. Disponível em: https://www.sciencedirect.com/science/article/abs/pii/ S0268401206000041?via\%3Dihub. Acesso em: 16 set. 2021.

SALO, Dorothea. Innkeeper at the Roach Motel. Library Trends, Baltimore, v. 57, n. 2, p. 98-123, 2008. DOI: https://doi.org/10.1353/lib.0.0031. Disponível em: https://muse.jhu.edu/article/262026. Acesso em: 16 set. 2021.

SHERPA. Institutional repositories: staff and skills set. [S. I.]: SHERPA, 2009. Disponível em: http://www. sherpa.ac.uk/documents/Staff and Skills Set 2009.pdf. Acesso em: 17 maio 2020.

SIMONS, Natasha; RICHARDSON, Joanna. New roles, new responsibilities: examining training needs of repository staff. Journal of Librarianship and Scholarly Communication, [s. I.], v. 1, n. 2, p. 1051, 2012. DOI: https://doi.org/10.7710/2162-3309.1051. Disponível em: https://www.iastatedigitalpress.com/llsc/article/ id/12680/. Acesso em: 16 set. 2021.

SUBER, Peter. Open access. Cambridge, MA: MIT Press, 2012. Disponível em: https://dash.harvard.edul bitstream/handle/1/10752204/9780262517638 Open Access.pdf?sequence=1. Acesso em: 15 set. 2021.

SWAN, Alma. Institutional repositories - now and next. In: DALE, Penny; BEARD, Jill; HOLLAND, Matt (ed.). University libraries and digital learning environments. Farnham: Ashgate Publishing, 2011. p. 119-134.

THOMAS, Wm. Joseph. The structure of scholarly communications within academic libraries. Serials Review, [s. I.], v. 39, n. 3, p. 167-171, 2013. DOI: https://doi.org/10.1016/j.serrev.2013.07.003. Disponível em: https:// www.sciencedirect.com/science/article/abs/pii/S0098791313000920. Acesso em: 16 set. 2021.

WICKHAM, Jackie. Repository management: an emerging profession in the information sector. Online Information, Londres: Olympia, 2010. Disponível em: https://nottingham-repository.worktribe.com/ output/1011665/repository-management-an-emerging-profession-in-the-information-sector. Acesso em: 17 dez. 2020.

ZUCCALA, Alesia; OPPENHEIM, Charles; DHIENSA, Rajveen. Managing and evaluating digital repositories. Information Research, v. 13, n. 1, 2008. Disponível em: http://informationr.net/ir/13-1/paper333.html. Acesso em: 16 set. 2021. 